\title{
Supporting Information: Titr-DMD - A Rapid, Coarse-Grained Quasi-All-Atom Constant pH Molecular Dynamics Framework
}

\author{
David J. Reilley, ${ }^{\dagger}$ Jian Wang, ${ }^{\ddagger}$ Nikolay V. Dokholyan, ${ }^{\ddagger, \S}$ Anastassia N. Alexandrova ${ }^{*,+, \|}$ \\ ${ }^{\dagger}$ Department of Chemistry and Biochemistry, University of California, Los Angeles, Los Angeles, California 90095-1569, \\ United States \\ ${ }^{\ddagger}$ Department of Pharmacology, Department of Biochemistry \& Molecular Biology, Penn State University College of \\ Medicine, Hershey, Pennsylvania 17033, United States \\ ${ }^{\S}$ Departments of Chemistry and Biomedical Engineering, Pennsylvania State University, University Park, Pennsylvania \\ 16802, United States \\ " California NanoSystems Institute, Los Angeles, California 90095-1569, United States
}

\section{Selection of Solvent Access Cutoff}

The exact value of the solvent access cutoff is system dependent and requires special attention/calibration. A cutoff of $75 \%$ was originally selected for all of the simulations conducted in this study. A Propka calculation on the initial structure for each system yielded buried values above $\sim 75 \%$ for only the entirely buried residues and those nestled in internal folds, while the external surface residues were all well below this percentage. Over the course of the Titr-DMD simulations, only 2 of the 40 residues with experimentally available $p K_{a}$ in the benchmark set stayed consistently buried (ASP26 in HTRX and CYS282 in HMCK), so these simulations were ultimately not too sensitive to the choice of solvent buried cutoff. This choice was, however, very critical for simulations of $p H$-conformational coupling, as they involved a study of whether the protonation state of a deeply buried residue (LYS66 in a staphylococcal nuclease mutant V66K) is coupled to the dynamics of the protein. In this case, we ran full simulations at a series of different solvent access cutoff (25\%, 35\%, 45\%, 55\%, and 65\%) and determined the most physical cutoff based on a comparison to the reduced surface SAS. LYS66 was then defined as solvent accessible if its amine group is within its van der Waals radius of three vertices (one face) of the SES. The comparison to the solvent access cutoff was done for a 20 ns test TitrDMD simulation of SNase V66K with a solvent access cutoff of $75 \%$. The solvent accessibility was then calculated for each

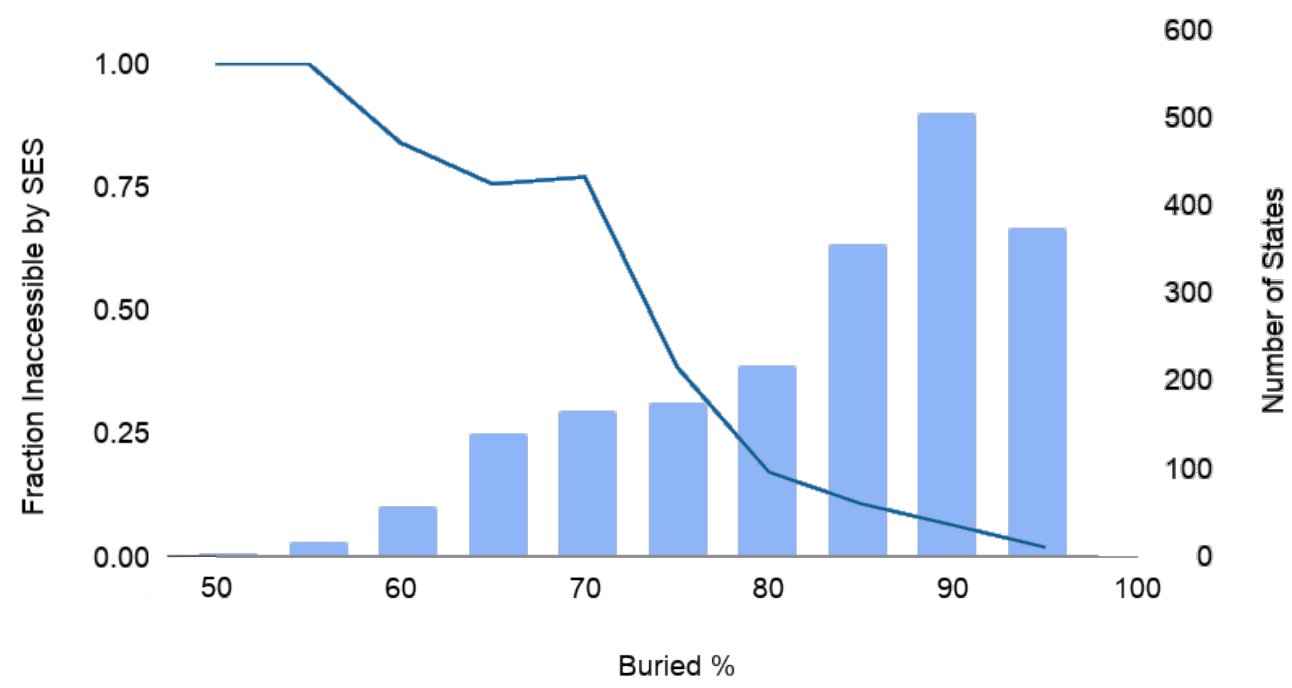

Figure S1: Comparison of the Propka buried percentage to solvent accessibility according the reduced surface SES. The dark blue line is the fraction of states that are solvent inaccessible according to the SES, where 1 is totally inaccessible and 0 is entirely accessible (left axis). The light blue histogram is the number of states in that bin (right axis). States are binned in units of 5 buried percentage points. Note that most states below $65-70 \%$ buried are solvent inaccessible according to the SES and that those below 55-60\% buried are entirely inaccessible according to the SES. 
iteration with a SAS generated by Chimera (v 1.13.1). ${ }^{1}$ The iterations were binned based on their Propka predicted percent buried, and the percent of states deemed to be solvent accessible was calculated for each bin (Figure S1). The results show that for SNase V66K there is a threshold around 65-75\% buried where LYS66 is generally buried above that point and not buried below that point. Structures do not, however, become entirely solvent accessible until below 55\%. It is important to note that such a low Propka buried \% is rare for LYS66 in our short test simulation. We therefore selected for analysis the SNase simulations with solvent access cutoffs of $65 \%$ and $45 \%$, one around the inflection point (in Figure S1) and one where LYS66 is only solvent accessible when it is assured based on our SES test. The results of the other cutoffs are reported later in this supporting information (Figure S3, Tables S1-S3).

\section{Additional Convergence Criterion for HMCK and SNase V66K}

Besides backbone RMSD and energy, convergence was also assessed in the HMCK and SNase V66K simulations by the $p K_{a}$ and frequency of (de)protonation of select residues (Figure S2). For HMCK, this residue was CYS283. A plot of the average Propka-predicted $p K_{a}$ over time for each replicate shows convergence on fixed values. Convergence was not checked based on the frequency of deprotonation of this residue, as the Propka $p K_{a}$ of this residue ended up consistently too high for deprotonation to occur at the $p H$ of the performed simulations. For SNase V66K, the tracked residue was LYS66. As with the RMSD and energy in the main text, these results represent the simulations at cutoffs of $65 \%$ and $45 \%$. The plot of the average Propka-predicted $p K_{a}$ over time for each replicate simulation shows convergence in a tight band of fixed values largely below 8. The plot of the frequency of LYS66 protonation shows distinct behavior between the $65 \%$ and $45 \%$ cutoff simulations. The upper band of trajectories that converge on full protonation are the $65 \%$ cutoff simulations, while the others are the $45 \%$ cutoff simulations. As the Propka $p K_{a}$ is higher than the $p H$ of all the simulations, the more solvent accessible $65 \%$ cutoff simulations converge on full protonation. However, the $45 \%$ cutoff simulations show some amount of protonation and deprotonation as well as divergence not resolved by the end of the simulation in some cases. Ultimately, this suggests that these simulations have captured an initial state of unraveling and the full, sustained unraveling occurs on a (likely significantly) longer timescale. The results can still be used to study the coupling between protonation of LYS66 and structures that lead to unraveling, which is discussed in the Results and Discussion section of the main text.
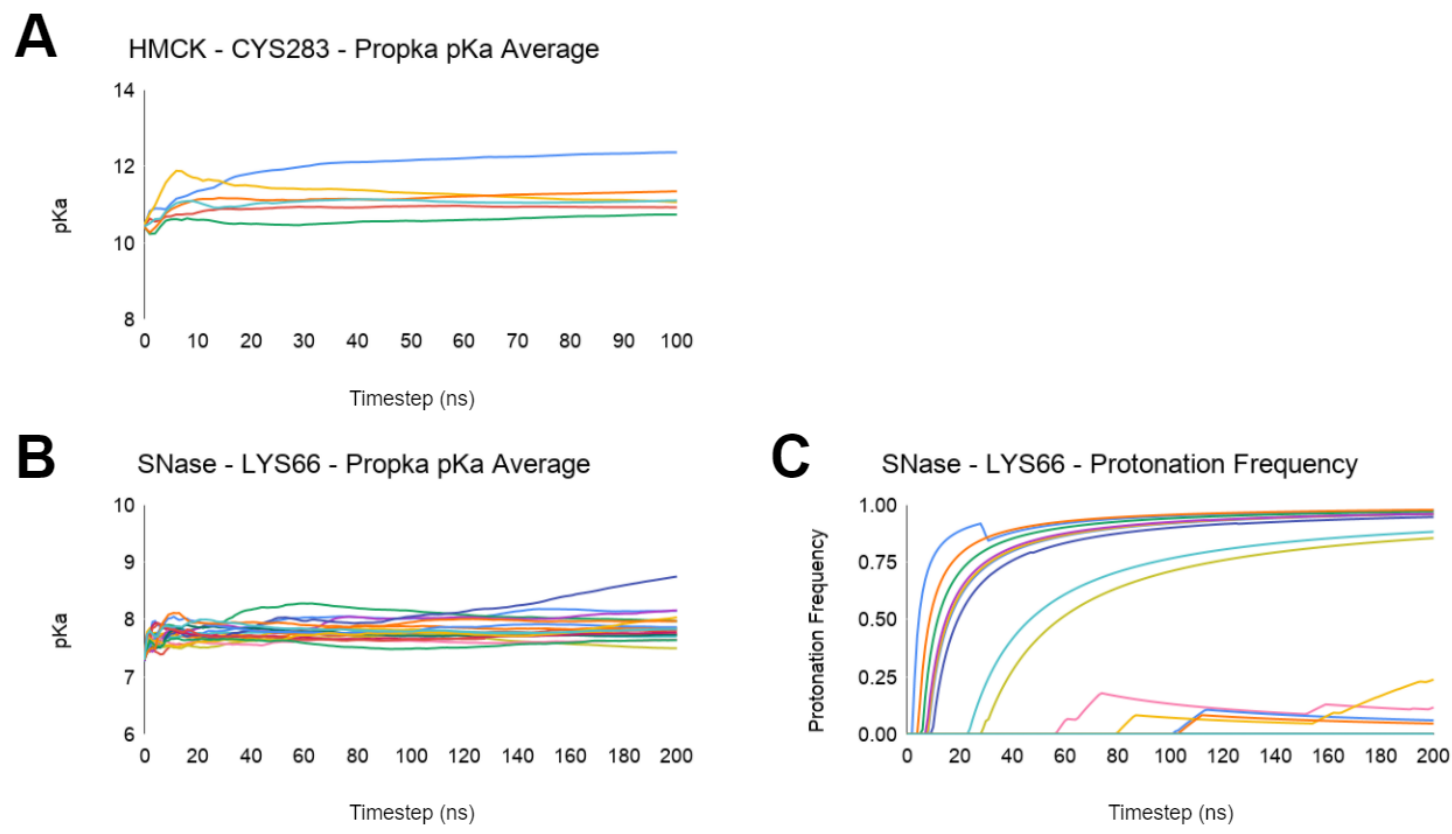

Figure S2: Average Propka-predicted $p K_{a}$ of (A) CYS283 in HMCK and (B) LYS66 in SNase V66K. The value at each timepoint is the average up to that point. The frequency of protonation of LYS66 (C) is also plotted. The protonation state is 0 for deprotonation to 1 for protonation. The protonation frequency at each timestep is the average of the protonation state up to that point. All HMCK simulations are plotted while only the $65 \%$ and $45 \%$ buried cutoff simulations are plotted for SNase V66K.

\section{Titr-DMD Simulations of SNase V66K at Other Cutoffs}

Titr-DMD simulations of the staphylococcal nuclease mutant V66K were also performed with solvent access cutoff values of $55 \%$, 35\%, and $25 \%$. All of the other settings for these simulations were the same as for the $45 \%$ and $65 \%$ cutoff simulations, as reported in the main text. These simulations achieved converged structures just like our others according to the metrics of backbone RMSD and corrected DMD potential energy (Figure S3A-B). The results of the 
other convergence criterion, average $p K_{a}$ and protonation frequency, are likewise similar to those of the $45 \%$ and $65 \%$ cutoff simulations (Figure S3C-D).
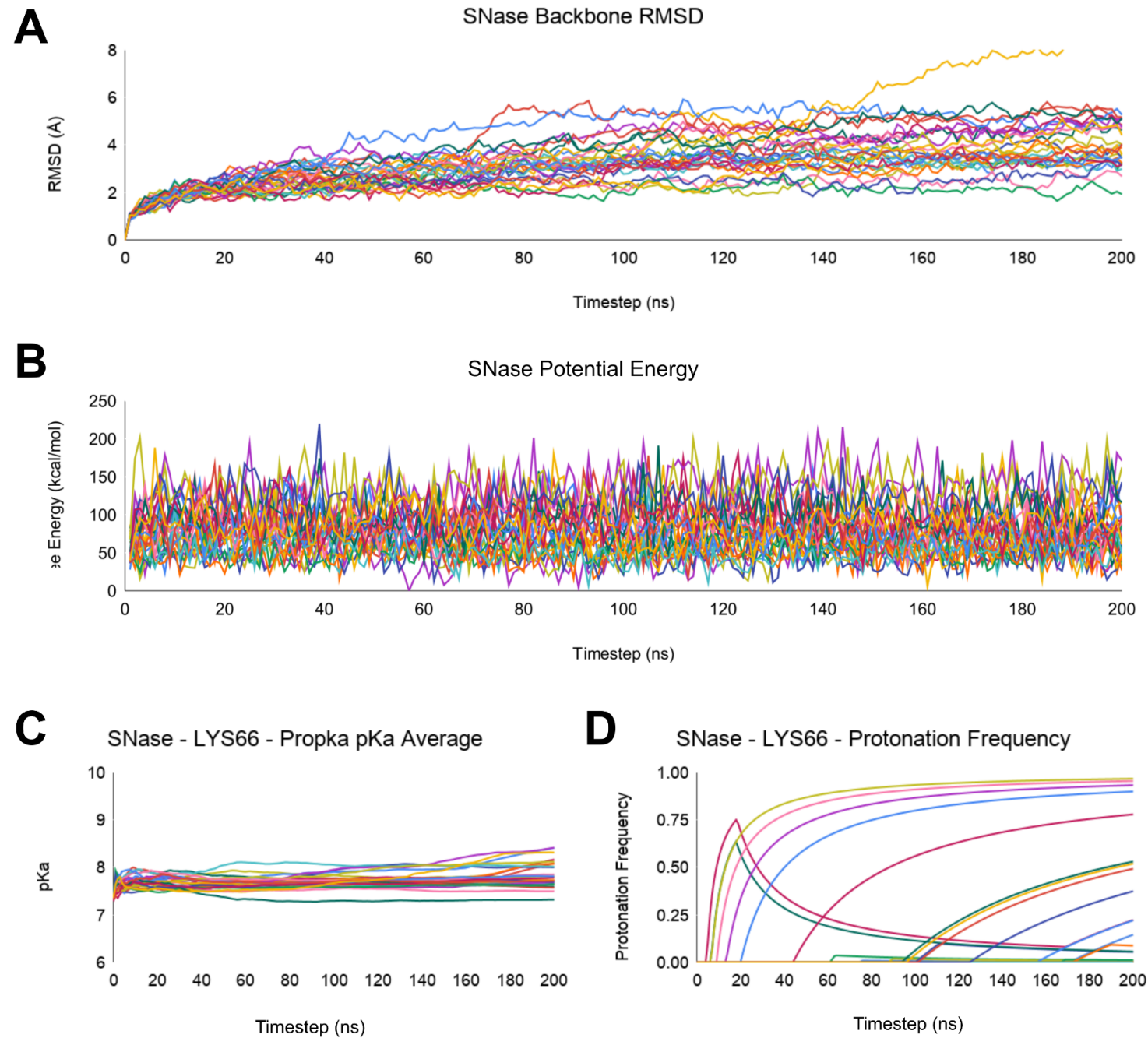

Figure S3: Convergence of Titr-DMD simulations of SNase V66K with solvent access cutoffs of 25\%, 35\%, and 55\%. Convergence is tracked by (A) the backbone RMSD, (B) the corrected DMD potential energy, (C) the average Propkapredicted pKa of LYS66, and (D) the protonation frequency of LYS66. For the protonation frequency, 0 represents deprotonation and 1 represents protonation. In both $(C)$ and $(D)$ the plotted point for each timestep is the average value up to that point. Nearly all simulations come to oscillate around fixed values by $200 \mathrm{~ns}$ for metrics A-C. The unconverged trajectories in metric D are for the 55\% solvent cutoff, showing intermediate behavior between the $65 \%$ and $45 \%$ simulations as the initial protonation takes a while. The average RMSD is $3.54 \pm 1.26 \AA$, while the average energy is $73.05 \pm 28.88 \mathrm{kcal} / \mathrm{mol}$ across all trajectories.

These simulations reinforce the $45 \%$ solvent access cutoff simulation as the most physically meaningful for SNase V66K pH-conformational dynamic coupling. As with the $45 \%$ and $65 \%$ cutoff simulations, the total percentage of iterations reporting an unraveled 65-69 loop across these simulations is much larger than the DMD simulations without titration and does not show any particular $p H$ dependent trend (Table S1). The iterations around protonation state changes show a consistently higher frequency of unraveling at $p H 5.7$ with most events at $p H 5.7$ coupled to some contemporaneous unraveling, consistent with the $45 \%$ and $65 \%$ cutoff simulations (Table S2). The only exception are the $25 \%$ cutoff simulations, which report $0 \%$ unraveling by event at $p H 5.7$ as few protonation and deprotonation events occur at all. The calculated $p K_{a}$ for LYS66 in these simulations are likewise consistent with the trend of the $45 \%$ and $65 \%$ cutoff simulations (Table S3). The Propka $p K_{a}$ are all off by the same amount, while the DMD $p K_{a}$ drop down to near the experimental value of 6.4. Note that for the 55\% cutoff simulation the DMD $p K_{a}$ is intermediate between those of the $65 \%$ and $45 \%$ simulations, which then steadies out in the $35 \%$ and $25 \%$ cutoff simulations. This suggests 
that a solvent access cutoff of $45 \%$ is the first to capture the experimental $p K_{a}$ of LYS66, making it the most physically meaningful solvent access cutoff for the SNase $\mathrm{pH}$-dependent dynamic behavior.

\begin{tabular}{|l|c|c|c|}
\hline Simulation & $\mathrm{pH} 4.6$ & $\mathrm{pH} 5.7$ & $\mathrm{pH} 7$ \\
\hline $25 \%$ cutoff & $5.99 \%$ & $4.15 \%$ & $5.19 \%$ \\
\hline $35 \%$ cutoff & $6.78 \%$ & $6.53 \%$ & $8.58 \%$ \\
\hline $55 \%$ cutoff & $7.88 \%$ & $6.43 \%$ & $7.01 \%$ \\
\hline
\end{tabular}

Table S1: Frequency of SNase V66K alpha helical loop 65-69 unraveling over the course of Titr-DMD simulations with solvent access cutoffs of 25\%, 35\%, and 55\%. These all report a much higher percentage of unraveled states than the $0.002 \%$ - $0.015 \%$ for DMD simulations without titration (found in Table 3 in the main text).

\begin{tabular}{|l|c|c|c|}
\hline Near Event & $\mathrm{pH} 4.6$ & $\mathrm{pH} 5.7$ & $\mathrm{pH} 7$ \\
\hline $25 \%$ cutoff & $10.98 \%$ & $0.00 \%$ & $7.25 \%$ \\
\hline $35 \%$ cutoff & $5.34 \%$ & $15.49 \%$ & $6.13 \%$ \\
\hline $55 \%$ cutoff & $31.33 \%$ & $10.67 \%$ & $24.09 \%$ \\
\hline By Event & & & \\
\hline $25 \%$ cutoff & $67 \%$ & $0 \%$ & $56 \%$ \\
\hline $35 \%$ cutoff & $71 \%$ & $93 \%$ & $63 \%$ \\
\hline $55 \%$ cutoff & $67 \%$ & $67 \%$ & $100 \%$ \\
\hline
\end{tabular}

Table S2: Frequency of unraveling of the SNase V66K 65-69 loop around LYS66 protonation and deprotonation events for simulations with solvent access cutoffs of 25\%, 35\%, and 55\%. As in Table 4 in the main text, 'Near event' refers to the percentage of iterations within 25 timesteps (before and after) of an event that are unraveled. This is only consistently elevated above the total simulation average in the $p H 5.7$ simulation. 'By event' refers to the percent of (de)protonation events that have at least one unraveled structure within 25 timesteps. This is also only consistently high for the $\mathrm{pH} 5.7$ simulations. The $25 \%$ cutoff simulation is the one outlier to this trend, reporting $0 \%$ for both forms of coupling, but that is likely due to the infrequency of (de)protonation events in those simulations.

\begin{tabular}{|l|c|c|}
\hline Simulation & DMD & Propka \\
\hline $25 \%$ cutoff & 5.78 & 7.78 \\
\hline $35 \%$ cutoff & 5.80 & 7.86 \\
\hline $55 \%$ cutoff & 6.32 & 7.89 \\
\hline
\end{tabular}

Table S3: Titr-DMD predicted $p K_{a}$ for LYS66 in the SNase mutant for simulations with 25\%, 35\%, and 55\% solvent access cutoffs. Note that the Propka $p K_{a}$ is consistently high across the cutoffs, while the DMD $p K_{a}$ drops down to a value close to the experimental $p K_{a}$ of 6.4 between the cutoffs of $55 \%$ and $35 \%$. 


\section{Computational Resource Scaling of Titr-DMD}

Full discussion of the resource scaling of Titr-DMD is found at the beginning of the Results and Discussion section of the main text. Plots of the computational cost benchmark calculations are found here (Figure S4).

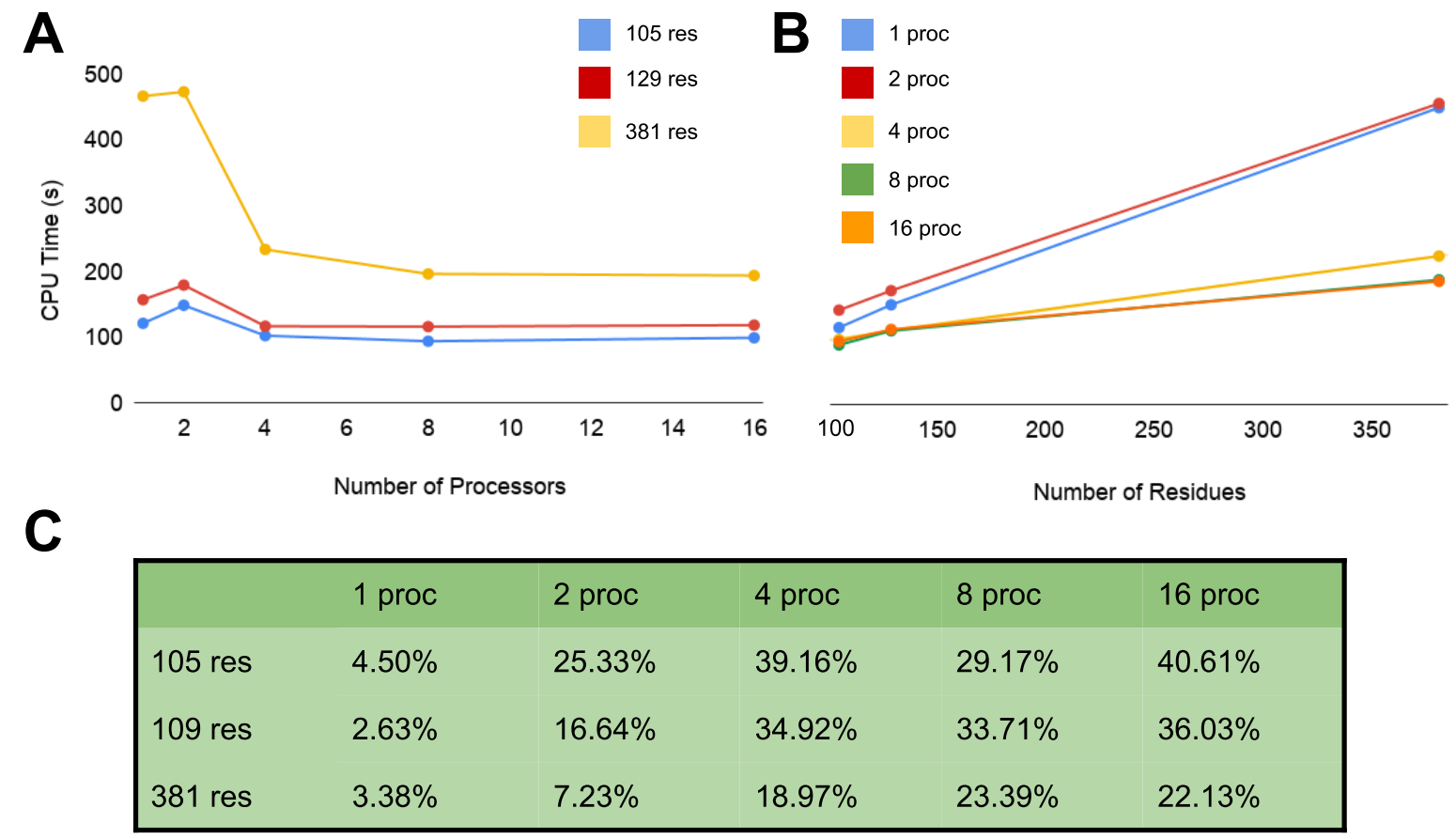

Figure S4: Computational resource scaling benchmark of Titr-DMD, plotted by (A) the number of processors and (B) the number of residues. Note the linear scaling with number of residues and that good performance is reached with four processors. (C) The percent increase of time for Titr-DMD over unmodified DMD. Note that the increase is relatively small and only becomes significant with many processors as the time DMD takes shortens.

\section{Comparison of Experimental and Calculated $p K_{a}$ Shifts from Null Values}

One issue with judging the accuracy of a CPHMD method using just the RMSE and MAE of all calculated $p K_{a}$ values is that it can be misleading if most of the experimental $p K_{a} \mathrm{~s}$ in the benchmarking dataset are close to solution values. This is known as a nondiscriminative benchmark (NDM), ${ }^{2}$ equally weighting these easier to predict points with ones that CpHMD methods tend to struggle with. Many CpHMD methods, including the Propka method used by Titr-DMD, are based on applying a calculated shift to a standard solution value for each amino acid. If most of the shifts of a dataset are small, then error from the method is often deceptively small as well and can average out the larger error from the few data points that deviate from the solution value significantly. Even the NULL model appears to perform well with such a dataset.

A more neutral test involves plotting the shift from solution of both the experimental and calculated $p K_{a}$ values against each other. The resulting plot provides easy visualization of points with large shifts and points where the experimental shift is not recapitulated well (Figure S5). The plot of our data for Titr-DMD from tables 1-4 demonstrates that TitrDMD recapitulates large $p K_{a}$ shifts in many cases, with a few notable outliers. The line of fit for our plot from linear regression has a slope of 0.95 - where 1 would represent perfect agreement with experiment on average. This value benefits some from error cancellation, but most points lie within about $1 \mathrm{pH}$ unit of the curve, including many with large shifts. We discuss the quality of Titr-DMD $p K_{a}$ predictions for different classes of amino acids at length already in the Results and Discussion section of the main text, but this plot further clarifies the observations from our data. 


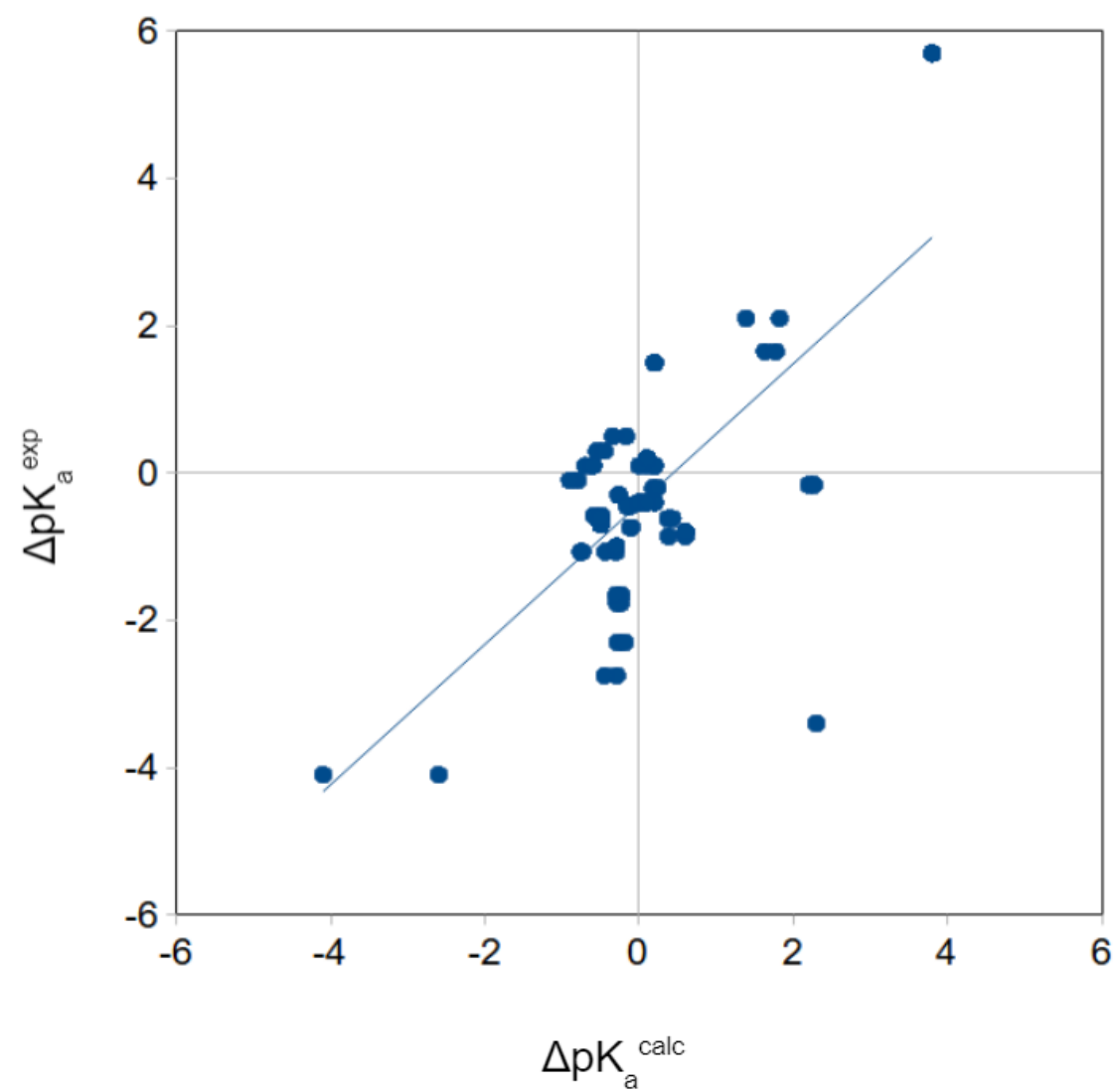

Figure S5: Plot of experimental and Titr-DMD calculated $p K_{a}$ shifts from solution values. Points include the DMD-averaged and Propka-averaged calculated values. A projected line of fit obtained from linear regression is also on the plot. Most points are within about $1 \mathrm{pH}$ unit of experiment, including many with large shifts. There are a few notable outliers, however. The point far from the origin in the lower right quadrant corresponds to the deeply buried HMCK CYS283. Many of the other points further from the line of fit and further from agreement with experiment are ASP and GLU residues in HEWL shifted to acidic values. The general absence of points far from the origin in the upper left and lower right quadrants demonstrates good overall qualitative agreement with experiment on shifts.

\section{References}

(1) Pettersen, E. F.; Goddard, T. D.; Huang, C. C.; Couch, G. S.; Greenblatt, D. M.; Meng, E. C.; Ferrin, T. E. UCSF Chimera-a Visualization System for Exploratory Research and Analysis. J. Comput. Chem. 2004, 25 (13), 1605-1612.

(2) Schutz, C. N.; Warshel, A. What Are the Dielectric "Constants" of Proteins and How to Validate Electrostatic Models? Proteins Struct. Funct. Bioinforma. 2001, 44 (4), 400-417. 